PROCEEDINGS OF THE

AMERICAN MATHEMATICAL SOCIETY

Volume 139, Number 6, June 2011, Pages 2217-2225

S 0002-9939(2010)10627-5

Article electronically published on November 10, 2010

\title{
DENDRITES AS POLISH STRUCTURES
}

\author{
RICCARDO CAMERLO
}

(Communicated by Julia Knight)

\begin{abstract}
It is shown that standard universal dendrites under the action of their group of homeomorphisms give rise to small Polish structures. Moreover, any non-singleton dendrite forming a small Polish structure (or, more generally, having at least one uncountable orbit) under the action of its group of homeomorphisms has $\mathcal{N} \mathcal{M}$-rank 1 . Finally, dendrites satisfy the existence of nm-independent extensions.
\end{abstract}

\section{INTRODUCTION AND BASIC NOTIONS}

In $\mathrm{K} 10$ the definition of a Polish structure is given as a pair $(X, G)$, where $G$ is a Polish group acting faithfully on the set $X$ in such a way that the stabilisers of singletons are closed.

If $(X, G)$ is a Polish structure and $A \subseteq X$, denote by $G_{A}$ the pointwise stabiliser of $A$. A Polish structure $(X, G)$ is small if for every $n \geq 1$ there are only countably many orbits of the action of $G$ on $X^{n}$. In particular, in an uncountable small Polish structure there are uncountable orbits.

The following is implicitly used in [K10].

Lemma 1. A Polish structure $(X, G)$ is small if and only if for any $a_{1}, \ldots, a_{n} \in X$, the action of $G_{\left\{a_{1}, \ldots, a_{n}\right\}}$ on $X$ has countably many orbits.

Proof. Suppose the action of $G_{\left\{a_{1}, \ldots, a_{n}\right\}}$ on $X$ has uncountably many orbits and let $K$ be a transversal for the orbit equivalence relation. Then all elements of $\left\{\left(a_{1}, \ldots, a_{n}, k\right)\right\}_{k \in K}$ are in different orbits of the action of $G$ on $X^{n+1}$.

Conversely, suppose the action of $G$ on some $X^{n+1}$ has uncountably many orbits and let $n$ be minimal with this property. If $n=0$, then there is nothing more to prove, so assume $n>0$. As the actions of $G$ on $X^{n}$ and on $X$ have countably many orbits, there are $a_{1}, \ldots, a_{n}, b \in X$ such that $G\left(a_{1}, \ldots, a_{n}\right) \times G b$ contains uncountably many orbits of the action of $G$ on $X^{n+1}$. Since each such orbit contains an element of the form $\left(a_{1}, \ldots, a_{n}, c\right)$, it follows that the action of $G_{\left\{a_{1}, \ldots, a_{n}\right\}}$ on $X$ has uncountably many orbits.

Though the definition of a Polish structure $(X, G)$ does not require $X$ to be a topological space, an important class of Polish structures is obtained when $X$ is a compact metric space and $G$ is the group of homeomorphisms of $X$ equipped with

Received by the editors December 8, 2009 and, in revised form, May 5, 2010; June 1, 2010; and June 4, 2010.

2010 Mathematics Subject Classification. Primary 03C45, 03E15, 54F15.

Key words and phrases. Small Polish structure, dendrite, universal dendrite.

(C)2010 American Mathematical Society

Reverts to public domain 28 years from publication 
the compact-open topology and acting on $X$ in the natural way. Among compact metric spaces, dendrites constitute some of the simplest examples: a dendrite is a compact, connected, locally connected metric space that does not contain simple closed curves. Definitions and basic properties about dendrites can be found in [N92]; those most needed in this paper are collected in section 2, for further reference.

This note investigates Polish structures of the form $(D, G)$, where $D$ is a dendrite and $G$ its group of homeomorphisms acting on $D$ in the natural way; when metric considerations will involve the group $G$, the supremum distance on $G$ is subsumed. A dendrite $D$ will be said to be small if the Polish structure $(D, G)$ is small.

Not all dendrites are small: let $D$ be a planar dendrite such that the set of branch points of $D$ is (] $0,1[\cap \mathbb{Q}) \times\{0\}$ with distinct branch points having different orders. Then all points in $[0,1] \times\{0\}$ lie in different orbits, so $D$ is not small. Notice that in this example there are uncountable orbits under homeomorphism, since $D$ contains open free arcs. For another example, let $D$ be a dendrite with a dense set of branch points, all of distinct order (such a dendrite can be obtained as in the construction in [N92, 10.37] of Ważewski's universal dendrite, but taking care that the branch points have pairwise different order). Then $D$ is rigid.

Let $(X, G)$ be a Polish structure, $\vec{a}=\left(a_{1}, \ldots, a_{n}\right) \in X^{n}$, and let $A \subseteq B$ be finite subsets of $X$. According to $\mathrm{K} 10$, we say that $\vec{a}$ is nm-dependent on $B$ over $A$ if $\left\{g \in G_{A} \mid g \vec{a} \in G_{B} \vec{a}\right\}$ is meagre in $G_{A}$; otherwise, $\vec{a}$ is nm-independent from $B$ over $A$. Using this, define a function $\mathcal{N} \mathcal{M}$ from the set of pairs $(\vec{a}, A)$, with $\vec{a}$ in some $X^{n}$ and $A$ a finite subset of $X$, to the ordinals satisfying $\mathcal{N} \mathcal{M}(\vec{a}, A) \geq \alpha+1$ if and only if there is a finite $B$ with $A \subseteq B \subseteq X$ such that $\vec{a}$ is nm-dependent on $B$ over $A$ and $\mathcal{N} \mathcal{M}(\vec{a}, B) \geq \alpha$. The $\mathcal{N} \mathcal{M}$-rank of $(X, G)$ is the supremum of all $\mathcal{N} \mathcal{M}(a, \emptyset)$, for $a$ ranging in $X$. Actually in K10 this definition is given only in the case of Polish structures admitting nm-independent extensions, to grant some good properties of $\mathcal{N M}$-ranks; the notation employed here differs slightly from the one used there.

In section 3 it will be shown that the so-called standard universal dendrites are small. Section 4 will establish that whenever $D$ is a dendrite with at least one uncountable orbit, then its $\mathcal{N} \mathcal{M}$-rank is 1 . In section 5 it will be proved that any dendrite $D$ admits nm-independent extensions: this means that for any $\vec{a} \in D^{n}$ and

finite subsets $A, B \subseteq D$ with $A \subseteq B$, there is $\vec{b} \in G_{A} \vec{a}$ such that $\vec{b}$ is nm-independent from $B$ over $A$.

\section{REVIEW OF DENDRITES}

For convenience, this section collects some definitions, properties and notation of dendrites that will be used in the sequel. A reference or a sketchy justification is also provided.

(1) For $D$ a dendrite, denote by $E(D)$ the set of its end points and by $R(D)$ the set of its branch points. This last set is countable for all dendrites (N92, Theorem 10.23]).

(2) The order of a point $x$ in $D$ will be denoted by $\operatorname{ord}(x, D)$. Then, $\operatorname{ord}(x, D) \leq$ $\aleph_{0}$ for any $x \in D($ N92, Corollary 10.20.1]).

(3) Since $D$ is arcwise connected and contains no simple closed curves, given $x, y \in D$ with $x \neq y$, there is a unique subarc of $D$ with end points $x, y$. It will be denoted by $A_{x y}^{D}$. 
(4) Every sequence of subdendrites of a dendrite pairwise meeting in at most one point has vanishing diameter. Otherwise, one could find a sequence of $\operatorname{arcs} A_{n}$, pairwise intersecting in at most one point, converging to an $\operatorname{arc} A$. By the condition on the $A_{n}$, the diameters of $A \cap A_{n}$ converge to 0 . Let $p, q$ be distinct points in $A$ and let $U, V$ be arcwise connected neighbourhoods of $p, q$, respectively, with diameters less than $\frac{1}{2} d(p, q)$. So there is $n$ with $U \cap A_{n} \neq \emptyset \neq V \cap A_{n}$ and at least one of $A_{n} \cap A \cap U, A_{n} \cap A \cap V$ is empty. But then the arc-connectedness of $U$ and $V$ yields at least two arcs joining $p$ and $q$.

(5) Every point $p$ in a dendrite $D$ has a neighbourhood basis whose members are dendrites whose boundaries in $D$ have finite cardinality. Indeed, by the regularity of $D$, there is an open neighbourhood basis of $p$ whose members have finite boundaries. By local connectedness, the connected components of open sets are open, so for each of such neighbourhoods consider the closure of the connected component containing $p$.

(6) If $C$ is a subdendrite of $D$, denote by $r_{C}: D \rightarrow C$ the first point map for $C([\mathbf{N 9 2}, \S 10.3])$.

\section{Standard universal DENDRites ARE SMALL}

Following CD94, if $J$ is a non-empty subset of $\{3,4, \ldots, \omega\}$ let $D_{J}$ be the unique (up to homeomorphism) dendrite such that:

- if $a \in R\left(D_{J}\right)$, then $\operatorname{ord}\left(a, D_{J}\right) \in J$;

- for any arc $I \subseteq D_{J}$ and any $n \in J$ there is $a \in I$ such that $\operatorname{ord}\left(a, D_{J}\right)=n$.

The dendrite $D_{J}$ has the following universality property: if $D$ is any dendrite such that $\forall x \in D \exists n \in J \operatorname{ord}(x, D) \leq n$, then there is a subset of $D_{J}$ homeomorphic to $D$. This section (Lemma 3 through Theorem 17) is intended to establish the following result.

Theorem 2. The Polish structure $\left(D_{J}, G\right)$, where $G$ is the group of homeomorphisms of $D_{J}$ acting on it in the natural way, is small.

To begin with, a standard back and forth argument gives the following.

Lemma 3. Let $U, V$ be arcs, with end points $a, b$ and $c, d$, respectively. Let $\left\{U_{n}\right\}_{n \in \mathbb{N}}$ and $\left\{V_{n}\right\}_{n \in \mathbb{N}}$ be pairwise disjoint countable dense subsets of $U \backslash\{a, b\}$ and $V \backslash\{c, d\}$, respectively. Then there is a homeomorphism $g: U \rightarrow V$ such that:

- $g(a)=c, g(b)=d$,

- $\forall n \in \mathbb{N} g\left(U_{n}\right)=V_{n}$.

Lemma 4. Let $a, d$ be distinct points of $D_{J}$ and $b, c \in A_{a d}^{D_{J}} \backslash\{a, d\}$ be such that $\operatorname{ord}\left(b, D_{J}\right)=\operatorname{ord}\left(c, D_{J}\right)$. Then there is a homeomorphism $\varphi: D_{J} \rightarrow D_{J}$ such that $\varphi(a)=a, \varphi(b)=c, \varphi(d)=d$.

Proof. Lemma 3 gives homeomorphisms $\zeta_{0}: A_{a b}^{D_{J}} \rightarrow A_{a c}^{D_{J}}, \zeta_{1}: A_{b d}^{D_{J}} \rightarrow A_{c d}^{D_{J}}$ such that $\zeta_{0}(a)=a, \zeta_{0}(b)=\zeta_{1}(b)=c, \zeta_{1}(d)=d$ and $\operatorname{ord}\left(x, D_{J}\right)=\operatorname{ord}\left(\zeta_{i}(x), D_{J}\right)$ for $i \in\{0,1\}, x \in d o m \zeta_{i}$. Let $\theta=\zeta_{0} \cup \zeta_{1}: A_{a d}^{D_{J}} \rightarrow A_{a d}^{D_{J}}$.

For each $u \in R\left(D_{J}\right) \cap A_{a d}^{D_{J}}$ there are either $\operatorname{ord}\left(u, D_{J}\right)-2$, if $\operatorname{ord}\left(u, D_{J}\right)$ is finite, or $\aleph_{0}$ connected components $\left\{F_{u n}\right\}_{n}$ of $D_{J} \backslash\{u\}$ disjoint from $A_{a d}^{D_{J}} ;$ moreover, each $D_{u n}=F_{u n} \cup\{u\}$ is homeomorphic to $D_{J}$ by [CD94, Theorem 6.2] and has $u$ as 
an end point. Fix a homeomorphism $\varphi_{u n}: D_{u n} \rightarrow D_{\theta(u) n}$ such that $\varphi_{u n}(u)=\theta(u)$ (its existence can again be justified by [CD94, Theorem 6.2]). Then define

$$
\varphi(x)=\left\{\begin{array}{lll}
\theta(x) & \text { if } \quad x \in A_{a d}^{D_{J}} \\
\varphi_{u n}(x) & \text { if } \quad x \in D_{u n}
\end{array}\right.
$$

Since for every $\varepsilon \in \mathbb{R}^{+}$all but finitely many $D_{u n}$ have diameter less than $\varepsilon$, function $\varphi$ is continuous.

Lemma 5. Let $a$ be an end point of $D_{J}$ and $b, c \in D_{J} \backslash\{a\}$ be such that neither of $A_{a b}^{D_{J}}, A_{a c}^{D_{J}}$ is a subarc of the other and $\operatorname{ord}\left(b, D_{J}\right)=\operatorname{ord}\left(c, D_{J}\right)$. Then there is a homeomorphism $\varphi: D_{J} \rightarrow D_{J}$ such that $\varphi(a)=a, \varphi(b)=c$.

Proof. As $a$ is an end point, $A_{a b}^{D_{J}} \cap A_{a c}^{D_{J}}$ is an arc. So, let $e \in D_{J}$ such that $A_{a b}^{D_{J}} \cap A_{a c}^{D_{J}}=A_{a e}^{D_{J}}$. Let $f, g$ be end points of $D_{J}$ such that $A_{a b}^{D_{J}} \subseteq A_{a f}^{D_{J}}, A_{a c}^{D_{J}} \subseteq A_{a g}^{D_{J}}$. Using Lemma 3, construct a homeomorphism $\theta: A_{e f}^{D_{J}} \rightarrow A_{e g}^{D_{J}}$ such that

$$
\theta(e)=e, \quad \theta(b)=c, \quad \forall x \in A_{e f}^{D_{J}} \operatorname{ord}\left(x, D_{J}\right)=\operatorname{ord}\left(\theta(x), D_{J}\right) .
$$

For each $u \in\left(\left(A_{e f}^{D_{J}} \cup A_{e g}^{D_{J}}\right) \cap R\left(D_{J}\right)\right) \backslash\{e\}$, let $\left\{F_{u n}\right\}_{n}$ be an enumeration of the connected components of $D_{J} \backslash\{u\}$ disjoint from $A_{a f}^{D_{J}} \cup A_{a g}^{D_{J}}$ and let $D_{u n}=F_{u n} \cup\{u\}$, which is homeomorphic to $D_{J}$. For $u \in\left(A_{e f}^{D_{J}} \cap R\left(D_{J}\right)\right) \backslash\{e\}$ fix homeomorphisms $\varphi_{u n}: D_{u n} \rightarrow D_{\theta(u) n}$ with $\varphi_{u n}(u)=\theta(u)$. Finally, define:

$$
\varphi(x)= \begin{cases}\theta(x) & \text { if } x \in A_{e f}^{D_{J}} \backslash\{e\}, \\ \theta^{-1}(x) & \text { if } x \in A_{e g}^{D_{J}} \backslash\{e\} \\ \varphi_{u n}(x) & \text { if } x \in D_{u n}, u \in\left(A_{e f}^{D_{J}} \cap R\left(D_{J}\right)\right) \backslash\{e\}, \\ \varphi_{u n}^{-1}(x) & \text { if } x \in D_{\theta(u) n}, u \in\left(A_{e g}^{D_{J}} \cap R\left(D_{J}\right)\right) \backslash\{e\}, \\ x & \text { otherwise. }\end{cases}
$$

Function $\varphi$ is a homeomorphism, similarly to the proof of Lemma 4 ,

Corollary 6. Let $X, Y$ both be homeomorphic to $D_{J}$. Let $a, b \in X, c, d \in Y$ such that $a \neq b, c \neq d, \operatorname{ord}(a, X)=\operatorname{ord}(c, Y), \operatorname{ord}(b, X)=\operatorname{ord}(d, Y)$. Then there is a homeomorphism $\varphi: X \rightarrow Y$ such that $\varphi(a)=c, \varphi(b)=d$.

Proof. Let $\left\{X_{n}\right\}_{n<o r d(a, X)},\left\{Y_{n}\right\}_{n<o r d(c, Y)}$ be enumerations of the connected components of $X \backslash\{a\}, Y \backslash\{c\}$, respectively, with $b \in X_{0}, d \in Y_{0}$. Set $H_{n}=X_{n} \cup$ $\{a\}, K_{n}=Y_{n} \cup\{c\}$ : these are all homeomorphic to $D_{J}$. Let $\varphi_{n}: H_{n} \rightarrow K_{n}$ be a homeomorphism such that $\varphi_{n}(a)=c$. By applying Lemma 4 or Lemma 5 , let $\psi: H_{0} \rightarrow H_{0}$ be a homeomorphism such that $\psi(a)=a, \psi(b)=\varphi_{0}^{-1}(d)$. Set $\varphi=\varphi_{0} \psi \cup \bigcup_{n>0} \varphi_{n}$. Then $\varphi$ is a homeomorphism, since for any $\varepsilon \in \mathbb{R}^{+}$the diameters of $H_{n}$ and $K_{n}$ are eventually less than $\varepsilon$.

Theorem 7. Let $A=\left\{a_{1}, \ldots, a_{n}\right\} \subseteq D_{J}$ and let $H=G_{A}$ be the group of homeomorphisms of $D_{J}$ fixing $a_{1}, \ldots, a_{n}$. Then the action of $H$ on $D_{J}$ has countably many orbits.

Proof. It can be assumed that $n \geq 2$. Let $T$ be the smallest subcontinuum of $D_{J}$ containing $a_{1}, \ldots, a_{n}$. So $T$ is a subtree of $D_{J}$; notice that $E(T) \subseteq A$. By enlarging $A$, if necessary, it can also be assumed that $R(T) \subseteq A$. Let $E_{1}, \ldots, E_{m}$ be subarcs of $D_{J}$ such that, letting $u_{l}, v_{l}$ be the end points of $E_{l}$ :

- $u_{l}, v_{l} \in A$ for all $l$; 
- each element of $A$ is an end point of some $E_{l}$;

- if $l \neq l^{\prime}$, then $E_{l}, E_{l^{\prime}}$ intersect at most at one of their end points;

- $T=\bigcup_{l=1}^{m} E_{l}$.

For $l \in\{1, \ldots, m\}$, let $F_{l}=E_{l} \backslash\left\{u_{l}, v_{l}\right\}$. The statement will be proved by establishing the following claim.

Claim. The orbits of $D_{J}$ under the action of $H$ are:

(0) each singleton in $A$;

(1) each set $\left\{x \in F_{l} \mid \operatorname{ord}\left(x, D_{J}\right)=k\right\}$ for $l \in\{1, \ldots, m\}, k \in\{2\} \cup J$;

(2) each set $\left\{x \in D_{J} \backslash\{a\} \mid r_{T}(x)=a, \operatorname{ord}\left(x, D_{J}\right)=k\right\}$, for $a \in A, k \in$ $\{1,2\} \cup J$

(3) each set $\left\{x \in D_{J} \backslash F_{l} \mid r_{T}(x) \in F_{l}, \operatorname{ord}\left(r_{T}(x), D_{J}\right)=h, \operatorname{ord}\left(x, D_{J}\right)=k\right\}$, for $l \in\{1, \ldots, m\}, h \in J, k \in\{1,2\} \cup J$.

Proof of claim. First notice that these sets are invariant under the action of $H$ and their union is $D_{J}$. It remains to show that for any pair $x, y$ of points in each of these, there is $\varphi \in H$ with $\varphi(x)=y$.

(1) If $x, y \in F_{l}$ are such that $\operatorname{ord}\left(x, D_{J}\right)=\operatorname{ord}\left(y, D_{J}\right)$, let $X=r_{T}^{-1}\left(F_{l}\right) \cup$ $\left\{u_{l}, v_{l}\right\}$. Notice that $X$ is homeomorphic to $D_{J}$, since each subarc of $X$ contains points of all orders in $J$. So the claim follows by applying Lemma 4 to find a homeomorphism $\psi$ of $X$ fixing $u_{l}, v_{l}$ and sending $x$ to $y$; then define $\varphi: D_{J} \rightarrow D_{J}$ as being equal to $\psi$ on $X$ and to the identity on $D_{J} \backslash r_{T}^{-1}\left(F_{l}\right)$ : this $\varphi$ is continuous by the glueing lemma.

(2) If $a \in A$, let $X=r_{T}^{-1}(\{a\})$, which (if not a singleton) is homeomorphic to $D_{J}$. Let $x, y \in X \backslash\{a\}$ be such that $\operatorname{ord}\left(x, D_{J}\right)=\operatorname{ord}\left(y, D_{J}\right)$. Use Corollary 6 to establish a homeomorphism $\psi: X \rightarrow X$ such that $\psi(a)=$ $a, \psi(x)=y$. Let $\varphi: D_{J} \rightarrow D_{J}$ agree with $\psi$ on $X$ and be the identity elsewhere.

(3) Let $x, y \in D_{J} \backslash F_{l}$ be such that

- $r_{T}(x), r_{T}(y) \in F_{l}$,

- $\operatorname{ord}\left(r_{T}(x), D_{J}\right)=\operatorname{ord}\left(r_{T}(y), D_{J}\right)$,

- $\operatorname{ord}\left(x, D_{J}\right)=\operatorname{ord}\left(y, D_{J}\right)$.

Applying Lemma 3, let $\theta: E_{l} \rightarrow E_{l}$ be a homeomorphism fixing the end points, such that $\forall z \in E_{l}, \operatorname{ord}\left(z, D_{J}\right)=\operatorname{ord}\left(\theta(z), D_{J}\right)$ and such that $\theta r_{T}(x)=r_{T}(y)$. For each $z \in\left(F_{l} \cap R\left(D_{J}\right)\right) \backslash\left\{r_{T}(x)\right\}$, fix a homeomorphism $\varphi_{z}: r_{T}^{-1}(\{z\}) \rightarrow r_{T}^{-1}(\{\theta(z)\})$ such that $\varphi_{z}(z)=\theta(z)$. Using Corollary 6, also let $\varphi_{r_{T}(x)}: r_{T}^{-1}\left(\left\{r_{T}(x)\right\}\right) \rightarrow r_{T}^{-1}\left(\left\{r_{T}(y)\right\}\right)$ be a homeomorphism with $\varphi_{r_{T}(x)}\left(r_{T}(x)\right)=r_{T}(y), \varphi_{r_{T}(x)}(x)=y$. Now define the bijection $\varphi: D_{J} \rightarrow D_{J}$ as follows:

$$
\varphi(u)=\left\{\begin{array}{lll}
u & \text { if } \quad u \notin r_{T}^{-1}\left(F_{l}\right) \\
\theta(u) & \text { if } \quad u \in F_{l}, \\
\varphi_{z}(u) & \text { if } \quad u \in r_{T}^{-1}\left(F_{l}\right) \backslash F_{l}, r_{T}(u)=z .
\end{array}\right.
$$

Again, by the vanishing of the diameters of the $r_{T}^{-1}(\{z\})$ the continuity of $\varphi$ follows. 


\section{RANKS OF DENDRITES}

Fix a dendrite $D$ and denote by $G$ its group of homeomorphisms. The goal of this section is to show that if $D$ has at least one uncountable orbit (in particular, if $D$ is small), then the $\mathcal{N} \mathcal{M}$-rank of $(D, G)$ is 1 .

Recall from [K10, Theorem 2.5(3)] that points $a \in \operatorname{Acl}(A)$, that is, points whose orbits are countable under the action of $G_{A}$ for some finite $A$, are nm-independent from $B$ over $A$ for any finite $B$ with $A \subseteq B$. Consequently, if the orbit of $a$ under $G$ is countable, then $\mathcal{N} \mathcal{M}(a, \emptyset)=0$. In particular, this holds for branch points of $D$. So it will be enough to compute $\mathcal{N} \mathcal{M}(a, \emptyset)$ when $a \in D$ is such that $\operatorname{ord}(a, D) \leq 2$.

Lemma 8. Let $(X, H)$ be any Polish structure, $\vec{a} \in X^{n}$ and let $A, B$ be finite subsets of $X$ with $A \subseteq B$. Suppose there is $i$ such that $H_{A} a_{i}$ is uncountable and $H_{B} a_{i}$ is countable. Then $\vec{a}$ is nm-dependent on $B$ over $A$. In particular $\mathcal{N} \mathcal{M}(\vec{a}, A) \geq 1$.

Proof. Let $H_{B} a_{i}=\left\{h_{0} a_{i}, h_{1} a_{i}, \ldots\right\}$ where each $h_{j}$ is in $H_{B}$. In order to show that $\left\{g \in H_{A} \mid g \vec{a} \in H_{B} \vec{a}\right\}$ is meagre in $H_{A}$, observe that

$$
\begin{aligned}
\left\{g \in H_{A} \mid g \vec{a} \in H_{B} \vec{a}\right\} & =\left\{g \in H_{A} \mid \vec{a} \in\left\{g^{-1} h_{0} \vec{a}, g^{-1} h_{1} \vec{a}, \ldots\right\}\right\} \\
& =\bigcup_{j}\left\{g \in H_{A} \mid \vec{a}=g^{-1} h_{j} \vec{a}\right\} \\
& =\bigcup_{j}\left\{g \in H_{A} \mid g^{-1} h_{j} \in H_{\left\{a_{1}, \ldots, a_{n}\right\}}\right\} \\
& =\bigcup_{j}\left(h_{j} H_{\left\{a_{1}, \ldots, a_{n}\right\}} \cap H_{A}\right) \\
& \subseteq \bigcup_{j}\left(h_{j} H_{\left\{a_{i}\right\}} \cap H_{A}\right) .
\end{aligned}
$$

Each term appearing in this last countable union, a coset of the stabiliser of $a_{i}$ in $H_{A}$, is closed and is nowhere dense in $H_{A}$, since the index of $H_{\left\{a_{i}\right\}} \cap H_{A}$ in $H_{A}$ is uncountable.

Lemma 9. Let $(X, H)$ be any Polish structure, $\vec{a} \in X^{n}$ and let $A, B$ be finite subsets of $X$ with $A \subseteq B$. If for all $i$ the orbit $H_{A} a_{i}$ is countable, then $\vec{a}$ is nm-independent from $B$ over $A$.

Proof. Notice that the hypothesis implies that $H_{A} \vec{a}$ is countable. So one can use the remark after [K10, Proposition 3.4] stating that [K10, Theorem 2.5] holds for imaginary extensions as well.

For convenience, however, the direct proof similar to $\mathrm{K} 10$, Theorem 2.5(3)] is as follows. The index of $H_{A \cup\left\{a_{1}, \ldots, a_{n}\right\}}$ in $H_{A}$ is countable, so $H_{A \cup\left\{a_{1}, \ldots, a_{n}\right\}}$ is nonmeagre in $H_{A}$. Consequently, $H_{B} H_{A \cup\left\{a_{1}, \ldots, a_{n}\right\}}$ is also non-meagre in $H_{A}$. Now apply [K10, Proposition 2.3].

Lemma 10. Let $a \in E(D)$ and let $B$ be a finite subset of $D$ with $a \notin B$. Then $\left\{g \in G \mid g(a) \in G_{B} a\right\}$ contains a neighbourhood of the identity; in particular, a is nm-independent from $B$ over $\emptyset$.

Proof. If $a$ is isolated in $G a$, let $\varepsilon \in \mathbb{R}^{+}$be such that there is no other point of $G a$ within $\varepsilon$ of $a$. Then $\left\{g \in G \mid g(a) \in G_{B} a\right\}$ contains the open sphere in $G$ centered in the identity and radius $\varepsilon$. So assume $a$ is not isolated in $G a$.

Let $T$ be the smallest subtree of $D$ containing $B$. Denote $p=r_{T}(a)$. Let $C$ be a subdendrite of $D$ such that $C$ is a neighbourhood of $a$ with diameter less than $d(a, p)$ and the boundary of $C$ in $D$ has exactly one element, say $q$. Then $q \in A_{a p}^{D}$. Pick $b \in E(C) \backslash\{a, q\}$; the existence of $b$ is granted by the fact that $a$ is not isolated in $G a$. Let $c=r_{A_{a q}^{D}}(b)$, call $L$ the connected component of $b$ in $D \backslash\{c\}$ and let $K=L \cup\{c\}$. Similarly, let $L^{\prime}$ be the connected component of $a$ in $D \backslash\{c\}$ and set 
$K^{\prime}=L^{\prime} \cup\{c\}$. Since $K$ is a neighbourhood of $b$ and $K^{\prime}$ is a neighbourhood of $a$, let $\varepsilon \in \mathbb{R}^{+}$be such that

- the open ball centered in $b$ and radius $\varepsilon$ is contained in $K$,

- the open ball centered in $a$ and radius $\varepsilon$ is contained in $K^{\prime}$,

- the open ball centered in $p$ and radius $\varepsilon$ is disjoint from $C$.

Fix any homeomorphism $f$ of $D$ less than $\varepsilon$ apart from the identity, in order to show $f(a) \in G_{B} a$. Notice that $f(b) \in K, f(a) \in K^{\prime}, f(p) \notin C$. Moreover, any arc having an end point in $K^{\prime}$ and the other in $D \backslash\left(K \cup K^{\prime}\right)$ has $c$ as a unique common point with $K$. So $A_{a p}^{D} \cap A_{b c}^{D}=\{c\}$; then $A_{f(a) f(p)}^{D} \cap A_{f(b) f(c)}^{D}=\{f(c)\}$, and $c \in A_{f(a) f(p)}^{D}$. Since $A_{f(a) f(p)}^{D}$ has an end point in $K^{\prime}$ and meets $A_{f(b) f(c)}^{D}$ in $f(c)$, this implies that $f(c)=c$. Consequently $f\left(K^{\prime}\right)=K^{\prime}$. So if $g: D \rightarrow D$ is defined as $f$ on $K^{\prime}$ and as the identity on $D \backslash K^{\prime}$, one has $g(a)=f(a), g \in G_{B}$, whence $f(a) \in G_{B} a$.

Corollary 11. If $a \in E(D)$ and the orbit of $a$ is uncountable, then $\mathcal{N} \mathcal{M}(a, \emptyset)=1$.

Proof. By Lemmas 8 and 10, for $B$ a finite subset of $D$, point $a$ is nm-dependent on $B$ over $\emptyset$ if and only if $a \in B$. Taken any finite $B \subseteq D$ with $a \in B$, by Lemma 9 , $a$ is nm-independent from $C$ over $B$ for any finite $C$ with $B \subseteq C$.

Lemma 12. Let $a \in D$ with ord $(a, D)=2$ and let $B$ be a finite subset of $D$ such that $a \notin B$. Then $\left\{g \in G \mid g(a) \in G_{B} a\right\}$ contains a neighbourhood of the identity. In particular, $a$ is nm-independent from $B$ over $\emptyset$.

Proof. By possibly enlarging $B$ it can be assumed that $B$ intersects both connected components of $D \backslash\{a\}$; say $B_{1}, B_{2}$ are such intersections. For $j \in\{1,2\}$ let $T_{j}$ be the smallest subtree of $D$ containing $B_{j}$ and set $p_{j}=r_{T_{j}}(a)$.

Case 1. There is a neighbourhood of $a$, of the form $A_{b c}^{D} \subseteq A_{p_{1} p_{2}}^{D}$, all of whose points have order 2 in $D$.

If $\varepsilon \in \mathbb{R}^{+}$is such that the $\varepsilon$-neighbourhood of $a$ is included in $A_{b c}^{D}$ and $f$ is a homeomorphism of $D$ less than $\varepsilon$ apart from the identity, let $a^{*}=f(a)$. Let $g$ be equal to the identity on $D \backslash A_{b c}^{D}$ and define $\left.g\right|_{A_{b c}^{D}}$ as a homeomorphism of $A_{b c}^{D}$ such that $g(b)=b, g(c)=c, g(a)=a^{*}$. Then $g \in G_{B}$ and thus $f(a) \in G_{B} a$.

Case 2. Point $a$ is the limit of a sequence of branch points of $D$ lying on $A_{p_{1} a}^{D}$, but there is $q \in A_{a p_{2}}^{D} \backslash\{a\}$ such that $A_{a q}^{D}$ does not contain any branch point of $D$ (or symmetrically, switching $\left.p_{1}, p_{2}\right)$. Pick $s, s^{\prime}, s^{\prime \prime}, r \in A_{p_{1} q}^{D} \backslash\left\{p_{1}, q\right\}$ such that

$$
A_{p_{1} s}^{D} \subset A_{p_{1} s^{\prime}}^{D} \subset A_{p_{1} a}^{D} \subset A_{p_{1} s^{\prime \prime}}^{D} \subset A_{p_{1} r}^{D} .
$$

Fix $\varepsilon \in \mathbb{R}^{+}$such that:

- the $\varepsilon$-neighbourhood of $p_{1}$ is included in $r_{A_{p_{1} p_{2}}^{D}}^{-1}\left(A_{p_{1} s}^{D}\right)$;

- the $\varepsilon$-neighbourhood of $a$ is included in $r_{A_{p_{1} p_{2}}^{D}}^{-1}\left(A_{s^{\prime} s^{\prime \prime}}^{D}\right)$;

- the $\varepsilon$-neighbourhood of $r$ is included in $A_{s^{\prime \prime} q}^{D_{1} p_{2}}$.

Let $f$ be a homeomorphism of $D$ less than $\varepsilon$ apart from the identity. By the choice of $\varepsilon, f(r) \in A_{s^{\prime \prime} q}^{D}$. Since $A_{a r}^{D}$ does not contain branch points, so $A_{f(a) f(r)}^{D}$ does not contain such points as well; once again using the choice of $\varepsilon, f(a) \in A_{a s^{\prime \prime}}^{D}$. Since points in $A_{a s^{\prime \prime}}^{D} \backslash\{a\}$ are not limits of a sequence of branch points, whereas $a$ is such a limit, the equality $f(a)=a$ is obtained. So $f(a) \in G_{B} a$. 
Case 3. Point $a$ is the limit of a sequence in $R(D) \cap A_{p_{1} a}^{D}$ and of a sequence in $R(D) \cap A_{a p_{2}}^{D}$.

Pick points $r_{1}, s, s^{\prime}, r_{2} \in A_{p_{1} p_{2}}^{D} \backslash\left\{p_{1}, p_{2}\right\}$ such that

$$
A_{p_{1} r_{1}}^{D} \subset A_{p_{1} s}^{D} \subset A_{p_{1} a}^{D} \subset A_{p_{1} s^{\prime}}^{D} \subset A_{p_{1} r_{2}}^{D}
$$

Let $\varepsilon_{1} \in \mathbb{R}^{+}$be such that:

- the $\varepsilon_{1}$-neighbourhood of $p_{j}$ is included in $r_{A_{p_{1} p_{2}}^{D}}^{-1}\left(A_{p_{j} r_{j}}^{D}\right)$, for $j \in\{1,2\}$;

- the $\varepsilon_{1}$-neighbourhood of $a$ is included in $r_{A_{p_{1} p_{2}}^{D}}^{-1}\left(A_{s s^{\prime}}^{D}\right)$.

For $j \in\{1,2\}$, pick $b_{j} \in A_{p_{j}}^{D} \cap R(D)$ with $d\left(a, b_{j}\right)<\varepsilon_{1}$ and take $c_{j} \in r_{A_{p_{1} p_{2}}^{D}}^{-1}\left(\left\{b_{j}\right\}\right) \backslash$ $\left\{b_{j}\right\}$. Let $\varepsilon_{2}<\varepsilon_{1}$ be such that the $\varepsilon_{2}$-neighbourhood of $c_{j}$ is contained in $r_{A_{p_{1} p_{2}}^{D}}^{-1}\left(\left\{b_{j}\right\}\right)$ and let $f$ be any homeomorphism of $D$ less than $\varepsilon_{2}$ apart from the identity. Then $f\left(b_{j}\right)=b_{j}$, since $A_{c_{j} b_{j}}^{D}=A_{p_{1} c_{j}}^{D} \cap A_{p_{2} c_{j}}^{D}$ and $A_{f\left(c_{j}\right) b_{j}}^{D}=A_{f\left(p_{1}\right) f\left(c_{j}\right)}^{D} \cap A_{f\left(p_{2}\right) f\left(c_{j}\right)}^{D}$. Consequently, $f\left(r_{A_{b_{1} b_{2}}^{D}}^{-1}\left(A_{b_{1} b_{2}}^{D} \backslash\left\{b_{1}, b_{2}\right\}\right)\right)=r_{A_{b_{1} b_{2}}^{D}}^{-1}\left(A_{b_{1} b_{2}}^{D} \backslash\left\{b_{1}, b_{2}\right\}\right)$. Let $g: D \rightarrow D$ be equal to the identity on $r_{A_{b_{1} b_{2}}^{D}}^{-1}\left(\left\{b_{1}, b_{2}\right\}\right)$ and to $f$ on $r_{A_{b_{1} b_{2}}^{D}}^{-1}\left(A_{b_{1} b_{2}}^{D} \backslash\left\{b_{1}, b_{2}\right\}\right)$. Then $g \in G_{B}, g(a)=f(a)$, granting $f(a) \in G_{B} a$.

Corollary 13. If $a \in D, \operatorname{ord}(a, D)=2$ and the orbit of $a$ is uncountable, then $\mathcal{N} \mathcal{M}(a, \emptyset)=1$.

Proof. As for Corollary 11, but using Lemmas 8, 12 and 9.

Corollary 14. Let $D$ be a dendrite. Then:

- if all orbits of $D$ are countable, then $\mathcal{N} \mathcal{M}(D)=0$;

- if there is an uncountable orbit in $D$, then $\mathcal{N} \mathcal{M}(D)=1$.

Proof. By Corollaries 11, 13 and the initial remark about points whose orbits are countable.

\section{ExistenCE OF INDEPENDENT EXTENSIONS}

One of the reasons for the importance of small Polish structures is that they satisfy the existence of nm-independent extensions: if the Polish structure $(X, H)$ is small, $\vec{a} \in X^{n}$, and $A, B$ are finite subsets of $X$ with $A \subseteq B$, then there exists $\vec{b} \in H_{A} \vec{a}$ such that $\vec{b}$ is nm-independent from $B$ over $A$. The proof of this is in K10, together with the discussion of its significance and examples of non-small Polish structures that admit (or do not admit) nm-independent extensions.

The situation for dendrites is that they do satisfy this property, even non-small ones. So this section is concerned with proving the following theorem, which exploits again arguments such as those in Lemmas 10 and 12.

Theorem 15. Let $D$ be a dendrite and $G$ its group of homeomorphisms. Then for all $\vec{a} \in D^{n}$, for all finite subsets $A, B \subseteq D$ with $A \subseteq B$, there is $\vec{b} \in G_{A} \vec{a}$ such that $\vec{b}$ is nm-independent from $B$ over $A$.

Proof. Given $\vec{a}, A, B$ as in the statement of the theorem, pick $\vec{b} \in G_{A} \vec{a}$ such that for each $i$, if $G_{A} a_{i}$ is uncountable, then $b_{i} \notin B$. The existence of $\vec{b}$ can be justified as follows: let $i_{0}$ be least such that $G_{A} a_{i_{0}}$ is uncountable but $a_{i_{0}} \in B$; then arbitrarily close to the identity there are elements of $G_{A}$ that move $a_{i_{0}}$. By finiteness of $B$, 
it is possible to pick $g \in G_{A}$ so that $g\left(a_{i_{0}}\right) \notin B$ and, if $a_{j} \notin B$, then $g\left(a_{j}\right) \notin B$. Now $g \vec{a}$ has at least one component less than $\vec{a}$ having uncountable $G_{A^{-}}$-orbit and belonging to $B$. Continuing this way, the tuple $\vec{b}$ is recovered. Now the aim is to show that $\vec{b}$ is nm-independent from $B$ over $A$.

Let $\varepsilon>0$ be less than all distances between pairwise distinct elements of $B \cup$ $\left\{b_{1}, \ldots, b_{n}\right\}$. By $(5)$ of section 2 , for each $i \in\{1, \ldots, n\}$ let $D_{i}$ be a dendrite such that

- $\operatorname{diam}\left(D_{i}\right)<\frac{\varepsilon}{2}$

- $D_{i}$ is a neighbourhood of $b_{i}$,

- the boundary of $D_{i}$ in $D$ is finite.

Let $B^{\prime}$ be the union of $B$ and the boundaries of all $D_{i}$ for $i \in\{1, \ldots, n\}$. Now notice that for all $i$ there is $\delta_{i}>0$ such that $\left\{g \in G_{A} \mid g\left(b_{i}\right) \in G_{B^{\prime}} b_{i}\right\}$ contains the $\delta_{i}$-neighbourhood in $G_{A}$ of the identity. Indeed, if $G_{A} b_{i}$ is countable (this includes the case $b_{i} \in B$ ), apply the proof of [K10, Theorem 2.5(3)]. If instead $G_{A} b_{i}$ is uncountable, then $\operatorname{ord}\left(b_{i}, D\right) \leq 2$; now apply either Lemma 10 or Lemma 12 to get a $\delta_{i}$-neighbourhood in $G$ of the identity contained in $\left\{g \in G \mid g\left(b_{i}\right) \in G_{B^{\prime}} b_{i}\right\}$ and thus the claim.

Let $\delta<\min \left(\delta_{1}, \ldots, \delta_{n}\right)$ be such that for each $i$ the $\delta$-ball centered in $b_{i}$ is contained in $D_{i}$. The proof of the theorem will be concluded by showing that for all $g \in G_{A}$, if $g$ is less than $\delta$ apart from the identity, then there is $h \in G_{B^{\prime}}$ with $h \vec{b}=g \vec{b}$. So fix such a $g$. Let $h_{i} \in G_{B^{\prime}}$ be such that $g\left(b_{i}\right)=h_{i}\left(b_{i}\right)$. Notice that $g\left(b_{i}\right) \in D_{i}$; thus $h_{i}$ is a homeomorphism of $D$ fixing all points of $B^{\prime}$ and sending $b_{i}$ to $g\left(b_{i}\right)$, both of these points being interior to $D_{i}$. This entails $h_{i}\left(D_{i}\right)=D_{i}$ : for one inclusion, if $p \in D_{i}$ was such that $h_{i}(p) \notin D_{i}$, then $A_{p b_{i}}^{D} \cap B^{\prime}=\emptyset$, while $h_{i}\left(A_{p b_{i}}^{D}\right) \cap h_{i}\left(B^{\prime}\right)=A_{h_{i}(p) h_{i}\left(b_{i}\right)}^{D} \cap B^{\prime} \neq \emptyset$; the other inclusion uses a similar argument on $h_{i}^{-1}$. It is now enough to define $h: D \rightarrow D$ by letting

$$
h(x)=\left\{\begin{array}{lll}
h_{i}(x) & \text { if } & x \in D_{i}, \\
x & \text { if } & x \notin \bigcup_{i=1}^{n} D_{i} .
\end{array}\right.
$$

\section{REFERENCES}

[CD94] W. J. Charatonik, A. Dilks, On self-homeomorphic spaces, Topology and its Applications 55 (1994), 215-238. MR.1259506 (95a:54054)

[K10] K. Krupiński, Some model theory of Polish structures, Transactions of the American Mathematical Society 362 (2010), 3499-3533. MR.2601598

[N92] S. B. Nadler, Jr., Continuum theory, Dekker, 1992. MR1192552 (93m:54002)

Dipartimento di Matematica, Politecnico di Torino, C.so Duca degli Abruzzi 24, 10129 TURin, ItALy

E-mail address: camerlo@calvino.polito.it 